\title{
SOME BIOLOGICAL PROPERTIES OF MYCOPHENOLIC ACID
}

\author{
Takao Noto, Mikio Sawada, Kunio Ando \\ and KenjiRo Koyama \\ Research Laboratories of Chugai Pharmaceutical Co., Ltd., \\ Toshima-ku, Tokyo, Japan
}

(Received for publication February 12, 1969)

\begin{abstract}
Mycophenolic acid (MA) inhibits the growth of some pathogenic fungi such as Candida albicans, Cryptococcus neoformans and several species of Trichophyton at low concentrations in vitro. The antibiotic is more effectvie against fungi at slightly acidic $\mathrm{pH}$ than neutral $\mathrm{pH}$. When administered to mice through the intraperitoneal, intramuscular and oral routes MA excreted to a few per cent in the urine. However, at no time was active MA detectable in the blood of mice. MA is effective against experimental trichophytosis of the guinea pigs, without noticeable skin irritation or inflammation.
\end{abstract}

Mycophenolic acid (MA) is an antifungal antibiotic found by Gosio ${ }^{1)}$ in 1896. Later, Glutterbuck and OXFord ${ }^{2)}$ reported that $\mathrm{MA}$ is produced by Penicillium brevi-compactum series to which Gosio's strain belonged. The structure of MA was elucidated by Birkinshaw ${ }^{3)}$ in 1952, as shown in Fig. 1. Although Alsbergat and FLOREY $^{5)}$ reported some biological properties of MA, detailed studies of the biological properties have not been made.

Recent findings that MA exerts significant antiviral and antitumor activities ${ }^{6,7)}$ prompted us to study some biological properties of the antibiotic. In this communication we describe in vitro antimicrobial properties, absorption and excretion in Fig. 1. Mycophenolic acid mice and the effect against experimental trichophytosis in the guinea pig.

\section{Materials and Methods}

Nystatin $(4,180 \mathrm{u} / \mathrm{mg})$ and griseofulvin were used as positive controls*.

The microorganisms used in this study are type cultures of our laboratory, including 54 strains of 25 species of fungi and 27 strains of 7 species of bacteria. The fungi were grown on malt extract agar (Difco, $\mathrm{pH} 5.2$ ) supplemented with $2 \%$ glucose. The medium for bacteria was MülleR-Hinton agar medium (Eiken, pH 7.2). For determination of minimum inhibitory concentration (MIC) a 2 -fold serial dilution method was used. The test organisms were inoculated by streaking to the assay media with a platinum loop from the cell suspensions of the test organisms (bacteria $10^{7.5}$ cells $/ \mathrm{ml}$, fungi $10^{6.5}$ cells $/ \mathrm{ml}$ ). The cultures were incubated $2 \sim 5$ days at $27^{\circ} \mathrm{C}$ for fungi and 18 hours at $37^{\circ} \mathrm{C}$ for bacteria.

Quantitative determination of MA; The thin-agar cylinder plate method was utilized for the quantitative determination of MA using Candida albicans as a test organism. The

* Nystatin and griseofulvin samples were kindly supplied by Sankyo Co., Ltd. and Nihon Kayaku Co., Ltd., respectively. 
cell suspension of the test organism was mixed with malt-dextrose agar medium. The cultures were incubated at $27^{\circ} \mathrm{C}$ for 24 hours. The zones of growth inhibitory formed by MA slowly disappeared from the margin with time, so it is necessary to incubate precisely for 24 hours. It is possible to determine MA in the range of $6 \sim 100 \mathrm{mcg} / \mathrm{ml}$ by this method.

Anti-trichophytosis activity of MA; MA was used as $5 \%$ solution in $50 \%$ ethanol and griseofulvin, the positive control, was used as $3 \%$ phenethyl alcohol solution. The hair was removed from back and both sides of guinea pigs, strain Hartley, weighing 300 $\pm 20 \mathrm{~g}$. After exfoliation with sand-paper on naked skin, the guinea pigs were infected with a spore suspension $\left(10^{6}\right.$ spores $\left./ \mathrm{ml}\right)$ of Trichophyton asteroides. The treatment was initiated 40 hours after infection, MA being given on the infected loci twice a day for 7 consecutive days. Anti-trichophytosis activity was determined by observing the symptoms caused by the infection on treated and untreated skin.

\section{Results and Discussions}

\section{Antimicrobial Activity of MA}

The antimicrobial spectra of MA are demonstrated in Tables 1 and 2. MA shows minimum inhibitory concentration (MIC) values of $3.9 \sim 31.2 \mathrm{mcg} / \mathrm{ml}$ against some pathogenic fungi such as Candida albicans, C. stellatoidea, C. tropicalis, C. parakrusei and Cryptococcus neoformans, whereas MA shows no activity against Candida guilliermondii, C. krusei, C. pseudotropicalis, Hausenula anomala and Saccharomyces cerevisiae. It is noteworthy that several species of Trichophyton were inhibited by relatively low

Table 1. Antifungal activity of mycophenolic acid and nystatin

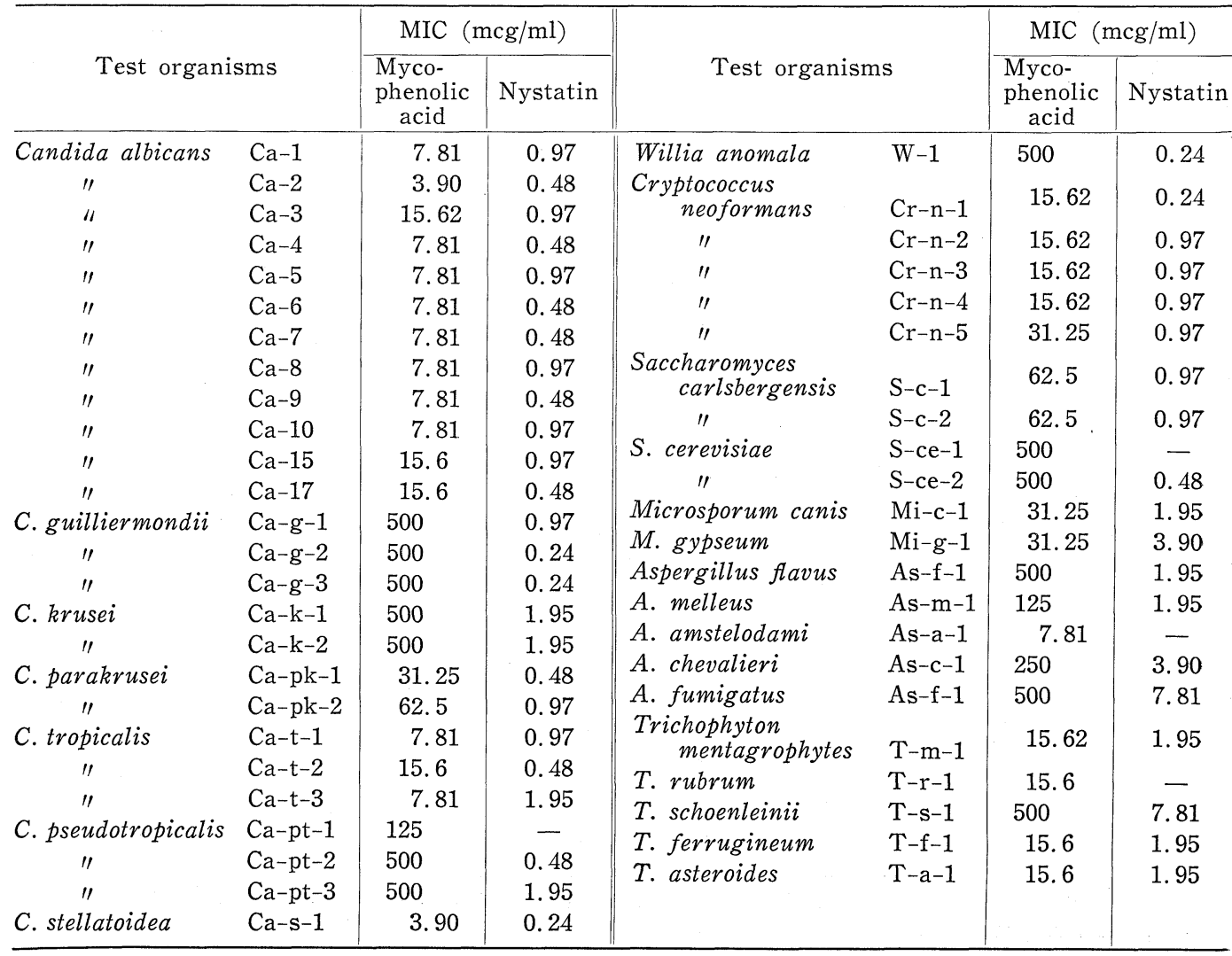


Table 2. Antibacterial activity of mycophenolic acid

\begin{tabular}{|c|c|c|c|c|c|}
\hline \multicolumn{2}{|c|}{ Test organisms } & \multirow{2}{*}{$\begin{array}{c}\begin{array}{c}\mathrm{MIC} \\
(\mathrm{mcg} / \mathrm{ml})\end{array} \\
31.25\end{array}$} & \multicolumn{2}{|l|}{ Test organisms } & \multirow{2}{*}{$\begin{array}{c}\begin{array}{c}\mathrm{MIC} \\
(\mathrm{mcg} / \mathrm{ml})\end{array} \\
125\end{array}$} \\
\hline Staphylococcus aureus & $209 \mathrm{P}$ & & Staphylococcus epidermidi. & $S-5$ & \\
\hline "1 & $209 \mathrm{P}(\mathrm{R})$ & 125 & Shigella flexneri & $2 \mathrm{a}$ & $>500$ \\
\hline "I & Terajima & 62.5 & 11 & $2 \mathrm{~b}$ & $>500$ \\
\hline$\prime \prime$ & 188 & 62.5 & Proteus vulgaris & $\mathrm{OX}-\mathrm{K}$ & 250 \\
\hline$\prime \prime$ & Kusama & 125 & 11 & $\mathrm{OX}-2$ & 500 \\
\hline$\prime \prime$ & B-5 & 31.25 & Escherichia coli & B-19 & $>500$ \\
\hline$" 1$ & B-8 & 31.25 & $\prime \prime$ & $\mathrm{C}-6$ & $>500$ \\
\hline "1 & B-9 & 125 & Pseudomonas aeruginosa & Ps-1 & $>500$ \\
\hline "l & B-15 & 31.25 & " & 1905 & $>500$ \\
\hline "I & B-19 & 31.25 & Salmonella enteritidis & S-1 & $>500$ \\
\hline " & B-43 & 125 & $\|$ & $\mathrm{S}-2$ & $>500$ \\
\hline " & B-46 & 125 & & & \\
\hline
\end{tabular}

Table 3. Effect of $\mathrm{pH}$ of medium on the activity of mycophenolic acid against various test organisms

\begin{tabular}{|c|c|c|c|c|c|c|c|}
\hline \multirow{2}{*}{\multicolumn{2}{|c|}{ Test organisms }} & \multicolumn{2}{|c|}{$\mathrm{MIC}(\mathrm{mg} / \mathrm{ml})$} & \multirow{2}{*}{\multicolumn{2}{|c|}{ Test organisms }} & \multicolumn{2}{|c|}{$\operatorname{MIC}(\mathrm{mg} / \mathrm{ml})$} \\
\hline & & \multirow{2}{*}{$\begin{array}{l}\mathrm{pH} \\
5.2 \\
6.25\end{array}$} & \multirow{2}{*}{$\begin{array}{c}\mathrm{pH} \\
7.3 \\
200\end{array}$} & & & & \\
\hline Candida albicans & $\mathrm{Ca}-3$ & & & Saccharomyces calsbergensis & S-c-1 & 200 & $>200$ \\
\hline "l & $\mathrm{Ca}-6$ & 6.25 & 200 & Microsporum canis & $\mathrm{Mi}-\mathrm{c}-1$ & 12.5 & 100 \\
\hline C. parakrusei & $\mathrm{Ca}-\mathrm{pk}-1$ & 12.5 & $>200$ & Aspergillus amstelodami & As-a-1 & 12.5 & 50 \\
\hline C. tropicalis & $\mathrm{Ca}-\mathrm{t}-1$ & 12.5 & $>200$ & Trichophyton mentagrophytes & $\mathrm{T}-\mathrm{m}-1$ & 6.25 & 25 \\
\hline C. stellatoidea & $\mathrm{Ca}-\mathrm{s}-1$ & 12.5 & 100 & T. asteroides & $\mathrm{T}-\mathrm{a}-1$ & 6.25 & 50 \\
\hline Cryptococcus neoformans & $\mathrm{Cr}-\mathrm{n}-1$ & 12.5 & 200 & & & & \\
\hline
\end{tabular}

Table 4. Effect of inoculum size on the activity of mycophenolic acid against various test organisms

\begin{tabular}{|c|c|c|c|c|c|}
\hline Test organisms & $\begin{array}{l}\text { Inoculum } \\
\text { size } \\
\text { (cells } / \mathrm{ml} \text { ) }\end{array}$ & $\begin{array}{c}\text { MIC } \\
(\mathrm{mcg} / \mathrm{ml})\end{array}$ & Test organisms & $\begin{array}{l}\text { Inoculum } \\
\text { size } \\
\text { (cells } / \mathrm{ml} \text { ) }\end{array}$ & $\begin{array}{c}\mathrm{MIC} \\
(\mathrm{mcg} / \mathrm{ml})\end{array}$ \\
\hline $\begin{array}{c}\text { Candida albicans } \\
\mathrm{Ca}-4\end{array}$ & $\begin{array}{l}10^{8} \\
10^{7} \\
10^{6} \\
10^{5}\end{array}$ & $\begin{array}{r}125 \\
15.6 \\
7.8 \\
3.9\end{array}$ & $\begin{array}{c}\text { Candida krusei } \\
\text { Ca-k-2 }\end{array}$ & $\begin{array}{l}10^{8} \\
10^{7} \\
10^{6} \\
10^{5}\end{array}$ & $\begin{array}{l}>125 \\
>125 \\
>125 \\
>125\end{array}$ \\
\hline $\begin{array}{c}\text { Candida parakrusei } \\
\text { Ca-pk-1 }\end{array}$ & $\begin{array}{l}10^{8} \\
10^{7} \\
10^{6} \\
10^{5}\end{array}$ & $\begin{array}{r}>125 \\
62.5 \\
31.2 \\
31.2\end{array}$ & $\begin{array}{l}\text { Candida } \\
\qquad \begin{array}{l}\text { guilliermondii } \\
\text { Ca-g-1 }\end{array}\end{array}$ & $\begin{array}{l}10^{8} \\
10^{7} \\
10^{6} \\
10^{5}\end{array}$ & $\begin{array}{r}>125 \\
>125 \\
>125 \\
\\
125\end{array}$ \\
\hline $\begin{array}{l}\text { Saccharomyces } \\
\text { carlsbergensis } \\
\text { S-c-2 }\end{array}$ & $\begin{array}{l}10^{8} \\
10^{7} \\
10^{6}\end{array}$ & $\begin{array}{r}>125 \\
125 \\
31.2\end{array}$ & $\begin{array}{l}\text { Candida } \\
\text { pseudotropicalis } \\
\quad \text { Ca-pt-3 }\end{array}$ & $\begin{array}{l}10^{8} \\
10^{7} \\
10^{6}\end{array}$ & $\begin{array}{l}>125 \\
>125 \\
>125\end{array}$ \\
\hline $\begin{array}{c}\text { Cryptococcus } \\
\text { neoformans. } \\
\text { Cr-n-3 }\end{array}$ & $\begin{array}{l}10^{8} \\
10^{7} \\
10^{6} \\
10^{5}\end{array}$ & $\begin{array}{c}125 \\
7.8 \\
7.8 \\
-\end{array}$ & $\begin{array}{l}\text { Willia anomala } \\
\qquad \text { W-1 }\end{array}$ & $\begin{array}{l}10^{8} \\
10^{7} \\
10^{6} \\
10^{5}\end{array}$ & $\begin{array}{l}>125 \\
>125 \\
>125 \\
>125\end{array}$ \\
\hline
\end{tabular}

concentration $(15.6 \mathrm{mcg} / \mathrm{ml})$. The antifungal spectrum of $\mathrm{MA}$ is specific and the activity is not as strong as nystatin which inhibited the growth of all fungi tested at concentrations of $0.2 \sim 7.8 \mathrm{mcg} / \mathrm{ml}$. 
MA shows moderate inhibition of Staphylococcus aureus but the other bacteria tested were insensitive. Most antifungal agents now used are highly toxic to mammals. It is interesting that the acute toxicity of MA is so low that mice could welltolerate a single intraperitoneal injection of $1,000 \mathrm{mg} / \mathrm{kg} .{ }^{7)}$

The MIC values of MA against sensitive fungi depends on $\mathrm{pH}$ of the assay media and the inoculum size of the test organisms (Tables 3 and 4). For example, several Candida albicans strains were inhibited at $6.3 \mathrm{mcg} / \mathrm{ml}$ at $\mathrm{pH} 5.2$ but grew normally at $\mathrm{pH} 7.3$ even in the presence of $200 \mathrm{mcg} / \mathrm{ml}$ of MA. MIC of MA for Cryptococcus neoformans was raised over 16 fold when the inoculum size increased from $10^{7}$ to $10^{8}$ cells/ml.

\section{Absorption and Excretion}

Table 5 shows that MA activity was recovered from urine after administration either intraperitoneally, intramuscularly or orally. Upon intramuscular administration of $250 \mathrm{mg} / \mathrm{kg}$ of MA urine levels of $42.5 \mathrm{mcg} / \mathrm{ml}$ were found after 30 minutes and $27.5 \mathrm{mcg} / \mathrm{ml}$ was still excreted in urine after 6 hours (Teble 5).
Table 5. Bio-assay values in urine after administration of mycophenolic acid to mice

\begin{tabular}{c|c|c|r|r|c}
\hline \multirow{2}{*}{$\begin{array}{c}\text { Dose } \\
(\mathrm{mg} / \mathrm{kg})\end{array}$} & \multirow{2}{*}{ Route } & \multicolumn{4}{|c}{ Urine concentration $(\mathrm{mcg} / \mathrm{ml})$} \\
\cline { 3 - 6 } & $\begin{array}{c}30 \\
\text { minutes }\end{array}$ & $\begin{array}{c}1 \\
\text { hour }\end{array}$ & $\begin{array}{c}3 \\
\text { hours }\end{array}$ & $\begin{array}{c}6 \\
\text { hours }\end{array}$ \\
\hline 250 & I M & 42.5 & 11.5 & 37.5 & 27.5 \\
250 & I P & 10.0 & 17.5 & 6.0 & - \\
500 & P O & 37.5 & 6.0 & 13.5 & 15.5 \\
\hline
\end{tabular}

The result is given by the mean of 5 animals.

Test organism : Candida albicans $\mathrm{Ca}-4$ However, no MA was detectable in the blood at any time post administration. This contradictory fact suggests that MA might be present in the blood bound with serum protein, showing no growth inhibitory activity. Although some MA was excreted in the urine, the total sum of recovered presented only a few per cent of the initial dose.

Effect against Experimental Trichophytosis

MA suppressed the symptoms caused by infection of Trichophyton asteroides without

Table 6. Effect of mycophenolic acid on experimental tricophytosis in guinea pigs

\begin{tabular}{|c|c|c|c|c|c|c|}
\hline Treatment & Dose & $\begin{array}{c}\text { Animal } \\
\text { No. }\end{array}$ & Curative rate* & Redness & Scale & Stimulus \\
\hline $\begin{array}{c}\text { None } \\
\text { (Control) }\end{array}$ & & $\begin{array}{l}1 \\
2 \\
3\end{array}$ & 0 & $\begin{array}{l}\text { H H } \\
H \text { H } \\
H+H\end{array}$ & $\begin{array}{l}H \text { H H } \\
H \text { H H } \\
H H\end{array}$ & \\
\hline $\begin{array}{r}\text { Griseofulvin } \\
(3 \% \text { soln. })\end{array}$ & $\begin{array}{c}0.2 \mathrm{ml} / \mathrm{part} \\
\text { b. i. d. }\end{array}$ & $\begin{array}{l}4 \\
5 \\
6\end{array}$ & $100 \%$ & $\begin{array}{l}--- \\
--- \\
---\end{array}$ & $\begin{array}{l}--- \\
--- \\
---\end{array}$ & $\begin{array}{l}--- \\
--- \\
---\end{array}$ \\
\hline $\begin{array}{c}\text { Mycophenolic } \\
\text { acid } \\
\text { (5\% soln.) }\end{array}$ & $\begin{array}{c}0.2 \mathrm{ml} / \mathrm{part} \\
\text { b. i. d. }\end{array}$ & $\begin{array}{l}7 \\
8 \\
9\end{array}$ & $\begin{array}{c}27.7 \% \\
16.6 \% \\
23.5 \% \\
(22.6 \% \text { in average })\end{array}$ & $\begin{array}{l}\perp+- \\
\perp \perp \perp \\
---\end{array}$ & $\begin{array}{l}--- \\
--- \\
---\end{array}$ & $\begin{array}{l}--- \\
--- \\
---\end{array}$ \\
\hline
\end{tabular}

* Guinea pigs were sacrificed after treatment to remove small pieces of infected skin (three pieces from back and both abdominal sides of each animal). Each small piece was divided equally into 6 portions to inoculate them onto SABouraud agar plates added with penicillin and streptomycin. The fungal growth was examined, after 1 -week incubation at $27^{\circ} \mathrm{C}$, and the curative rate was calculated as follows :

$$
\text { Curative rate }(\%)=\frac{\left(\begin{array}{l}
\text { Total number } \\
\text { of skin pieces }
\end{array}\right)-\left(\begin{array}{l}
\text { Number of skin pieces } \\
\text { indicated fungal growth }
\end{array}\right)}{\text { Total number of skin pieces }} \times 100(\%)
$$


irritation and inflammation to the skin (Table 6). When small pieces of treated skin were incubated at $27^{\circ} \mathrm{C}$ for 7 days on a suitable medium, $77.4 \%$ of the pieces possessed viable $T$. asteroides. This fact indicated that $\mathrm{MA}$ is a fungistatic agent rather than a fungicidal one. Since the symptoms caused by the proliferation of the fungus were suppressed, the growth of the organism was inhibited as long as MA was present, but once MA was removed from the treated skin, the fungus began to grow again. Thus, MA shows moderate inhibitory activity against experimental trichophytosis of guinea pigs without adverse side-effects. However, usefulness will be limited because of the fungistatic nature of the compound.

\section{Acknowledgement}

The authors express their sincere thanks to Dr. T. AкIвA, Director of Research Laboratories of Chugai Pharmaceutical Co., Ltd., for his advices during the study and in preparing the manuscript.

\section{References}

1) Gosio, B. : Ricerche bacteriologiche e chimiche sulle alterazioni del mais. Rivista Igiene Sanita Publica Ann. $7: 825 \sim 868$, 1896 quoted from Florey, H. W.; K. Gilliver, M. A. Jennings \& A. G. Sanders: Mycophenolic acid. An antibiotic from Penicillium brevi-compactum Dierckx. Lancet 1946-1:46 49, 1946

2) Clutterbuck, P. W.; A. E. Oxford, H. Raistrick \& G. Smith : Studies in the biochemistry of micro-organisms. The metabolic products of the Penicillium brevi-compactum series. Biochem. J. $26: 1441 \sim 1458,1932$

3) Birkinshaw, J. H.; H. Raistrick \& D. J. Ross : Studies in the biochemistry of micro-organisms. The molecular constitution of mycophenolic acid, a metabolic product of Penicillium brevicompactum Dierckx. III. Further observations on the structural formula from mycophenolic acid. Biochem. J. $50: 630 \sim 634,1952$

4) Alsberg, C. L. \& O. F. Black : Contribution to the study of maize deterioration. U. S. Dept. Agr. Plant Industry Bull. $270: 7 \sim 48,1913$

5) Florey, H. W.; K. Gilliver, M. A. Jennings \& A. G. Sanders : Mycophenolic acid. An antibiotic from Penicillium brevi-compactum Dierckx. Lancet 1946-1:46 49, 1946

6) Ando, K.; S. Suzuki, G. Tamura \& K. Arima : Antiviral activity of mycophenolic acid. Studies on antiviral and antitumor antibiotics. IV. J. Antibiotics $21: 649 \sim 652,1968$

7) Williams, R. H.; D. H. Lively, D. C. Delong, J. C. Chine, M. J. Sweeney, G. A. Poore \& S. H. LARSEn : Mycophenolic acid. Antiviral and antitumor properties. J. Antibiotics $21: 463 \sim 464$, 1968 